\title{
Incidence of postoperative atrial fibrillation in transdermal $\beta$-blocker patch users is lower than that in oral $\beta$-blocker users after cardiac and/ or thoracic aortic surgery
}

\author{
Homare Okamura $^{1}$ D $\cdot$ Mamoru Arakawa $^{1} \cdot$ Atsushi Miyagawa $^{1} \cdot$ Hideo Adachi $^{1}$
}

Received: 4 December 2018 / Accepted: 23 April 2019 / Published online: 2 May 2019

(C) The Japanese Association for Thoracic Surgery 2019

\begin{abstract}
Objective Postoperative atrial fibrillation (POAF) after open heart surgery is associated with a high risk of mortality and morbidity. Although oral $\beta$-blockers are usually recommended to prevent POAF, the efficacy of a transdermal $\beta$-blocker patch in preventing POAF is unclear. We compared the incidence of POAF between users of oral and transdermal bisoprolol. Methods We investigated 108 patients who underwent cardiac and/or thoracic aortic surgery between April 2016 and February 2018. We compared perioperative clinical and hemodynamic variables between 49 patients treated with a transdermal bisoprolol patch and 59 patients treated with an oral bisoprolol fumarate.

Results POAF occurred in $24 \%$ of patients in the transdermal and in $46 \%$ of patients in the oral bisoprolol groups $(p=0.027)$. No intergroup difference was observed in in-hospital mortality, perioperative blood pressures and heart rates, and other morbidities. Multivariable logistic regression analysis revealed that the use of transdermal bisoprolol was independently associated with a lower rate of POAF (odds ratio $0.21,95 \%$ confidence interval $0.05-0.84, p=0.027$ ).

Conclusions A transdermal bisoprolol patch is an effective and safe $\beta$-blocker drug delivery system. The incidence of POAF in this group was lower than that in users of oral bisoprolol.
\end{abstract}

Keywords Atrial fibrillation $\cdot \beta$-blocker · Cardiac surgery

\section{Introduction}

Postoperative atrial fibrillation (POAF) occurs in 13-40\% of patients after open heart surgery and is associated with prolonged hospitalization, stroke, increased economic costs, and mortality [1-4]. Oral $\beta$-blockers are recommended to treat POAF after cardiac and thoracic surgery [5]. The 2017 European guidelines recommend perioperative oral $\beta$-blocker therapy as a Class IIa indication to prevent POAF [6]. In contrast, Japanese guidelines recommend statins to prevent POAF after cardiac surgery [7]. The effectiveness of antiarrhythmic drugs other than $\beta$-blockers to prevent POAF is not well established as class IIb recommendation in the Japanese guideline [7].

Homare Okamura

homareo@jadecom.jp

1 Department of Cardiovascular Surgery, Nerima Hikarigaoka Hospital, 2-11-1 Hikarigaoka, Nerima-Ku, Tokyo 179-0072, Japan
The transdermal patch comprising the selective $\beta 1$-blocker bisoprolol (Bisono ${ }^{\circledR}$ Tape; Toa Eiyo, Tokyo, Japan) became commercially available in Japan in 2013. The first-pass effect is lower and drug bioavailability is higher with the transdermal administration of bisoprolol than that observed with oral administration of the drug [8]. Phase 2 clinical trials have shown that plasma concentrations of bisoprolol remained more steady with a lesser tendency to show spikes in concentration with the use of a transdermal bisoprolol patch (TBP) than with oral bisoprolol [9]. Godo et al. showed lower peak and higher trough concentrations of bisoprolol in users of the transdermal patch than that in users of the oral preparation [10]. Its efficacy in preventing POAF as well as hypotension and bradycardia is attributable to the stabilized blood concentration of bisoprolol available in the transdermal patch.

In this study, we compared the incidence of POAF between users of TBP and oral bisoprolol fumarate (OBF) in patients undergoing cardiac and/or thoracic aortic surgery. 


\section{Methods}

\section{Patients and methods}

We performed a retrospective study based on our hospital database. Between April 2016 and February 2018, a total of 216 patients underwent cardiac and/or thoracic aortic surgery at Nerima Hikarigaoka Hospital. We excluded patients with a history of atrial fibrillation, those not using postoperative $\beta$-blockers, those who did not undergo cardiopulmonary bypass, those who underwent endovascular treatment, and those with perioperative extracorporeal membrane oxygenation use. Eventually, 108 patients were included in this study (Fig. 1). We routinely prescribed bisoprolol for the prevention of POAF postoperatively (if $\beta$-blocker use was not contraindicated). Between April 2016 and April 2017, patients were administered OBF, and after May 2017, patients were administered TBP instead of OBF. In this study period, the indication for surgery and perioperative patient care remained unchanged. We compared preoperative patient characteristics and in-hospital outcomes between 59 patients administered OBF and 49 patients administered TBP to evaluate differences in the effect of $\beta$-blockers. Patients' preoperative characteristics and intraoperative variables in both groups are shown in Tables 1 and 2. POAF was defined as the new onset of postoperative atrial fibrillation lasting $>30$ s recorded using a telemetry system throughout hospitalization. Average observation period identical to postoperative hospital stay was $23 \pm 14$ days (median 19 days). When POAF occurred, nurses reported to a doctor who finally confirmed the diagnosis of POAF. Blood pressure and heart rate were measured preoperatively, upon admission to the intensive care unit, and postoperatively. Measurements later than postoperative day (POD) 1 were performed at 6 a.m. Good pain control was achieved using oral or intravenous

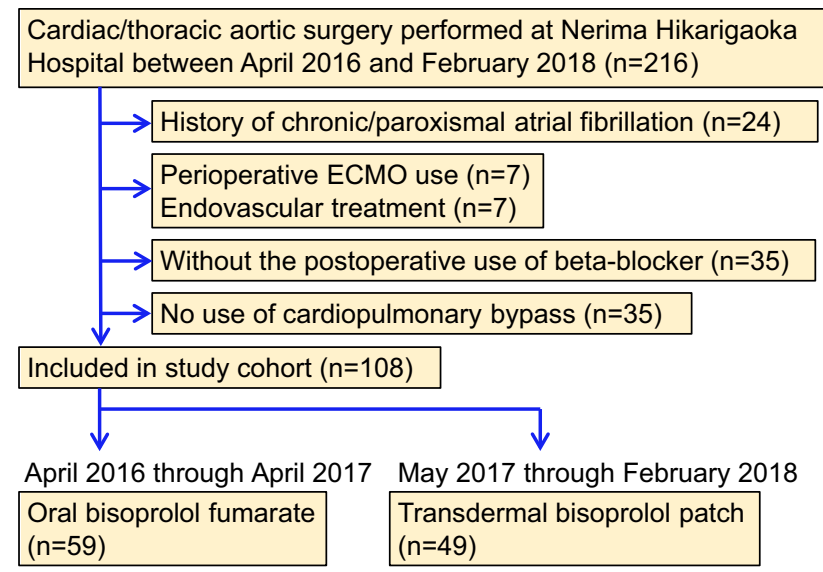

Fig. 1 Flow diagram shows the study population. ECMO extracorporeal membrane oxygenation analgesia. Other anti-arrhythmic agents including landiolol were postoperatively used for the treatment of any arrhythmia, not before POAF occurred, in 17 patients (29\%) in the OBF group and in 8 patients (16\%) in the TBP group without significant intergroup difference $(p=0.170)$. Postoperative arrhythmia except for atrial fibrillation was basically treated with amiodarone. Thoracic epidural analgesia was not used. Follow-up data including survival and the incidence of atrial fibrillation were obtained via a patient chart review. The mean follow-up period was $16 \pm 9$ months. The Institutional Review Board of our hospital approved the use of patient data for this retrospective study.

\section{Administration of $\beta$-blocker}

The administration of $\beta$-blocker commenced on POD 1 and was continued throughout admission. The OBF dosage was $2.5 \mathrm{mg}$ per day, and TBP dosage was $4 \mathrm{mg}$ (equivalent to $2.5 \mathrm{mg}$ of OBF) [11]. The dosage of $\beta$-blocker was adjusted based on age, cardiac function, renal function blood pressure, and heart rate in individual patient. As a result, a total of 34 patients (32\%) had lower dosage of $\beta$-blocker.

The dosage of bisoprolol was down-titrated in 22 patients (37\%) in the OBF group and 12 patients $(25 \%)$ in the TBP group without significant difference $(p=0.212)$. The mean $\%$ dosages to desirable dosage (i.e., actual mean dosage $/ 4 \mathrm{mg}$ in the transdermal group and actual mean dosage $/ 2.5 \mathrm{mg}$ in the oral group) were $86.2 \pm 23.4 \%$ in the transdermal group and $81.8 \pm 39.6 \%$ in the oral group, respectively $(p=0.471)$. The rates of down-titration or discontinuation of bisoprolol during hospitalization were 51\% (30 patients) in the OBF group and 39\% (19 patients) in the TBP group without significant intergroup difference $(p=0.247)$. The $\beta$-blockers were administered at 7 a.m. If hypotension (systolic blood pressure $<90 \mathrm{mmHg}$ ) or bradycardia (heart rate $<60$ beats per minute) occurred, the $\beta$-blocker was down-titrated or discontinued. Other $\beta$-blockers were not used concomitantly. Medications other than $\beta$-blockers that had been administered preoperatively were re-initiated once oral intake was possible.

\section{Statistical analysis}

The data in this study were expressed as mean \pm standard deviation for continuous variables and as frequencies and percentages for categorical variables. Patient characteristics and echocardiographic data were compared between groups using the Fisher's exact test for categorical variables and the unpaired Student's $t$ test for continuous variables. Prolonged vasopressor administration was defined as use of vasopressor later than postoperative day 1 . High dose of diuretics was defined as postoperative use of oral furosemide of $40 \mathrm{mg}$ or more daily. 
Table 1 Preoperative patient characteristics per study group

\begin{tabular}{llll}
\hline Characteristic & $\begin{array}{l}\text { Transdermal group } \\
(n=49)\end{array}$ & Oral group $(n=59)$ & $p$ value \\
\hline Age (years) & $69.2 \pm 14.5$ & $68.4 \pm 14.0$ & 0.759 \\
Male sex & $25(51)$ & $25(42)$ & 0.440 \\
Body mass index $\left(\mathrm{kg} / \mathrm{m}^{2}\right)$ & $22.3 \pm 3.7$ & $23.2 \pm 4.7$ & 0.283 \\
NYHA class 3 or 4 & $17(35)$ & $23(39)$ & 0.613 \\
Hypertension & $37(76)$ & $33(56)$ & 0.043 \\
Diabetes mellitus & $4(8)$ & $10(17)$ & 0.251 \\
Dyslipidemia & $9(18)$ & $13(22)$ & 0.811 \\
Ischemic heart disease & $9(18)$ & $10(17)$ & 1.000 \\
Stroke & $6(12)$ & $5(8)$ & 0.541 \\
Chronic obstructive pulmonary disease & $2(4)$ & $1(2)$ & 0.589 \\
Chronic kidney disease & & $7(12)$ & 0.751 \\
Hemodialysis & $4(8)$ & $3(5)$ & 1.000 \\
Left ventricular ejection fraction $(\%)$ & $2(4)$ & $62.0 \pm 13.9$ & 0.865 \\
Left atrial diameter (mm) & $61.4 \pm 12.3$ & $40 \pm 7$ & 0.274 \\
Brain natriuretic peptide (pg/ml) & $37 \pm 9$ & $282 \pm 491$ & 0.684 \\
Preoperative medication & $330 \pm 607$ & & 1.000 \\
ACE inhibitor & & $8(14)$ & 0.389 \\
Angiotensin II receptor blocker & $7(14)$ & $18(31)$ & 0.847 \\
Calcium blocker & $11(22)$ & $24(41)$ & 0.376 \\
Statin & $21(43)$ & $9(15)$ & 0.159 \\
$\beta$-blocker & $10(20)$ & $4(7)$ & 0.687 \\
Aldosterone blocker & $13(27)$ & &
\end{tabular}

Values are number of patients $(\%)$ or mean $\pm \mathrm{SD}$

${ }^{\text {a }}$ Serum creatinine $>1.5 \mathrm{mg} / \mathrm{dl}$

NYHA New York Heart Association, ACE angiotensin converting enzyme

\begin{tabular}{llll}
\hline Variable & $\begin{array}{l}\text { Transdermal group } \\
(n=49)\end{array}$ & Oral group $(n=59)$ & $p$ value \\
\hline Type of surgery & & & \\
Isolated valve surgery & $19(39)$ & $21(36)$ & 0.842 \\
Isolated CABG & $1(2)$ & $2(3)$ & 1.000 \\
Valve surgery +CABG & $4(8)$ & $4(7)$ & 1.000 \\
Isolated aortic surgery & $23(47)$ & $29(49)$ & 0.849 \\
Aortic surgery + valve surgery & $1(2)$ & 0 & 0.454 \\
Others & $1(2)$ & $3(5)$ & 0.625 \\
Cardiopulmonary bypass time (min) & $157 \pm 51$ & $143 \pm 57$ & 0.191 \\
Aortic cross-clamp time (min) & $104 \pm 38$ & $101 \pm 38$ & 0.704 \\
\hline
\end{tabular}

Values are number of patients $(\%)$ or mean $\pm \mathrm{SD}$

$C A B G$ coronary artery bypass grafting
Logistic regression analysis was performed to identify predictors of POAF. Variables included age, sex, body mass index, the New York Heart Association class 3/4, hypertension, diabetes, dyslipidemia, ischemic heart disease, stroke, chronic obstructive pulmonary disease, chronic kidney disease (serum creatinine level $>1.5 \mathrm{mg} / \mathrm{dl}$ ), hemodialysis, left ventricular ejection fraction, left atrial diameter, brain natriuretic peptide level, preoperative medication used, type of surgical procedure performed, and use of TBP. Variables with $p$ values $<0.25$ using univariate analysis were subjected to multivariable logistic regression analysis. Overall survival after discharge was estimated using the Kaplan-Meier method, and comparisons between two groups were performed with log-rank test. All analyses were performed 
using the SPSS software version 25 (IBM Corp., Armonk, NY, USA). A $p$ value $<0.05$ was considered statistically significant.

\section{Results}

\section{Patient characteristics and operative data in the groups administered transdermal and the oral bisoprolol}

As shown in Table 1, the likelihood of hypertension in patients administered TBP was higher than that in patients administered OBF. No intergroup difference was observed in variables including age and renal dysfunction, which are reportedly associated with the incidence of POAF. Preoperative medication use did not differ between groups. Operative data are shown in Table 2. No intergroup differences were observed in type of surgical procedures, cardiopulmonary bypass, and aortic cross-clamp times.

\section{Clinical outcomes}

Early outcomes are presented in Table 3. Overall hospital mortality was $0 \%$ in the transdermal and $3.4 \%$ in the oral group, and this difference was statistically non-significant. Among the two deaths in the oral group, one patient had POAF. The incidence of POAF was 24.5\% (12 patients) in the transdermal and $45.8 \%$ (27 patients) in the oral group. Among all cases, the rates of POAF was 35\% (14/40) in isolated valve surgery, $33 \%(1 / 3)$ in isolated coronary artery bypass grafting (CABG), 35\% (18/52) in isolated aortic surgery, $63 \%(5 / 8)$ in valve surgery and CABG, $0 \%$ $(0 / 1)$ in valve and aortic surgery, and 25\% (1/4) in others. Regarding the timing of onset of POAF, the median interval between surgery and the onset of POAF was 4 days, and this intergroup difference was statistically non-significant. Among patients with POAF, 69\% (27 patients) developed POAF within 5 days postoperatively. In our study, the treatments for POAF included intravenous amiodarone, pilsicainide or landiolol. No intergroup difference was observed in stroke rates, re-exploration for bleeding, prolonged ventilator use, and intensive care unit stay. As shown in Table 3, no intergroup difference was noted in the rates of prolonged vasopressor administration, use of temporary pacing, high dose of diuretics and discontinuation of $\beta$-blocker. Regarding the effect of discontinuation/tapering of $\beta$-blocker on the incidence of POAF, the rates of POAF were similar between the normal dose and discontinuation/tapering dose groups (normal dose vs. discontinuation/tapering dose, 41\% (24/59) vs. $31 \%(15 / 49), p=0.318)$. In 16 patients who discontinued $\beta$-blocker, POAF occurred in $25 \%(2 / 8)$ in the transdermal group and in 63\% (5/8) in the oral group without significant intergroup difference $(p=0.315)$.

\section{Blood pressure and heart rate}

Blood pressures and heart rates were compared preoperatively, upon admission to the intensive care unit, and on PODs 1, 2, 3, 5, and 7. No intergroup differences were observed in blood pressures and heart rates.

\section{Predictors of postoperative atrial fibrillation}

We assessed the independent risk factors for POAF using a multivariable logistic regression model. Multivariable analysis showed that the use of TBP was an independent predictor for reduced occurrence of POAF (odds ratio $0.21,95 \%$ confidence interval $0.05-0.84, p=0.027$ ). Other predictors included coronary artery bypass grafting, and hypertension, as shown in Table 4.

\begin{tabular}{llll}
\hline Variable & $\begin{array}{l}\text { Transdermal group } \\
(n=49)\end{array}$ & Oral group $(n=59)$ & $p$ value \\
\hline In-hospital mortality & $0(0)$ & $2(3)$ & 0.500 \\
New onset of atrial fibrillation & $12(24)$ & $27(46)$ & 0.027 \\
Stroke & $2(4)$ & $2(3)$ & 1.000 \\
Re-exploration for bleeding & 0 & 0 & 1.000 \\
Prolonged ventilator use $>48 \mathrm{~h}$ & $11(22)$ & $16(27)$ & 0.658 \\
Prolonged vasopressor administration & $17(35)$ & $13(22)$ & 0.195 \\
Use of temporary pacing & $7(14)$ & $9(15)$ & 1.000 \\
Use of high dose of diuretics & $7(14)$ & $14(24)$ & 0.235 \\
Discontinuation of $\beta$-blocker & $8(16)$ & $8(14)$ & 0.788 \\
Intensive care unit stay (day) & $4.5 \pm 2.5$ & $5.4 \pm 3.1$ & 0.132 \\
\hline
\end{tabular}

Values are number of patients $(\%)$ or mean \pm SD 
Table 4 Predictors of postoperative atrial fibrillation

\begin{tabular}{|c|c|c|c|c|}
\hline \multirow[t]{2}{*}{ Variable } & \multicolumn{2}{|l|}{ Univariate } & \multicolumn{2}{|l|}{ Multivariable } \\
\hline & OR $(95 \% \mathrm{CI})$ & $p$ value & OR $(95 \% \mathrm{CI})$ & $p$ value \\
\hline Coronary artery bypass grafting & $2.33(0.66-8.20)$ & 0.188 & $11.46(1.7-78.3)$ & 0.013 \\
\hline Hypertension & $2.42(0.99-5.86)$ & 0.051 & $4.81(1.0-22.2)$ & 0.044 \\
\hline Use of transdermal bisoprolol patch & $0.38(0.17-0.88)$ & 0.024 & $0.21(0.05-0.84)$ & 0.027 \\
\hline Left atrial size $(\mathrm{mm})$ & $1.07(0.99-1.15)$ & 0.074 & $1.09(0.99-1.20)$ & 0.082 \\
\hline Body mass index $\left(\mathrm{kg} / \mathrm{m}^{2}\right)$ & $1.11(1.00-1.22)$ & 0.042 & $1.11(0.95-1.30)$ & 0.201 \\
\hline Age (years) & $1.02(0.99-1.05)$ & 0.140 & - & - \\
\hline Male sex & $0.61(0.27-1.35)$ & 0.221 & - & - \\
\hline
\end{tabular}

$O R$ odds ratio, $C I$ confidence interval

\section{Follow-up data}

Overall survival was similar between the groups at average 16 months of follow-up $(p=0.485)$. One-year survival was $100 \%$ in the transdermal group and $94.6 \%$ in the oral group. Paroxysmal atrial fibrillation occurred in 1 patient $(2.0 \%)$ of the transdermal group and in 2 patients $(3.4 \%)$ of the oral group at follow-up $(p=1.000)$. Of them, one patient in the oral group underwent catheter ablation 3 months after surgery.

\section{Discussion}

The principal finding in this study was that a TBP was associated with lower rates of POAF in patients undergoing cardiac and/or thoracic aortic surgery. Regarding negative inotropic effects, postoperative blood pressure and heart rate were similar between the TBP and the OBF groups.

Reportedly, several risk factors including advanced age, hypertension, obesity, low left ventricular ejection fraction, renal dysfunction, and left atrial volume are associated with POAF after open heart surgery [2, 12-14]. Among various risk factors, activation of the sympathetic nervous system plays an important role in the pathophysiology of POAF. Sympathetic stimulation increases intracellular calcium, which promotes automaticity and triggered activity. Increased sympathetic activity prior to onset of $\mathrm{AF}$ has been observed [5]. $\beta$-blocker prevents POAF by blocking sympathetic tone. Yokota et al. showed that preoperative use of $\beta$-blocker was an independent predictor of POAF [15]. However, in our study, preoperative use of $\beta$-blocker was not associated with the incidence of POAF. Multivariable analysis in our study showed that non-use of the transdermal patch, coronary artery bypass grafting, and hypertension were independent predictors of POAF.

Reportedly, perioperative administration of oral $\beta$-blockers prevents POAF after open heart surgery [16-18]. The American College of Cardiology Foundation/American Heart Association guidelines recommend the use of oral $\beta$-blockers and amiodarone for the prevention of POAF after coronary artery bypass grafting [19]. However, regarding routine use of amiodarone for prevention of POAF, we had concerns about adverse effects of amiodarone. Hillis et al. showed that POAF typically occurs within 5 days postoperatively, with a peak incidence on POD 2 [19]. In our cohort, $69 \%$ of POAF cases occurred within 5 days postoperatively. No intergroup difference was observed in the timing of POAF.

Several studies have reported the effectiveness of intravenously administered $\beta$-blockers for the treatment and prevention of POAF. Sakamoto et al. showed that the conversion of POAF to sinus rhythm in patients administered landiolol (a short-acting intravenously administered $\beta$-blocker) was more common and quicker than that in patients administered diltiazem. Additionally, the risk of heart failure related to landiolol was lesser than that related to diltiazem [20]. Sezai et al. showed that 48-h administration of intravenous landiolol in patients undergoing coronary artery bypass grafting prevented POAF [21]. They also reported that landiolol prevented POAF in patients with left ventricular dysfunction undergoing cardiac surgery [22]. Their study showed that landiolol was associated with lower levels of brain natriuretic peptide and a shorter hospital stay, without requiring discontinuation of the drug for hypotension or bradycardia [22]. Perioperative intravenous administration of $\beta$-blockers has been shown to be effective and safe in the prevention of POAF. However, cost-effectiveness of intravenous $\beta$-blockers remains a concern. In addition, the superiority of intravenous $\beta$-blocker over oral $\beta$-blocker has not been clarified in terms of the prevention of POAF.

Several reports in the literature describe the efficacy and safety of a TBP in patients with hypertension and heart failure. Momomura et al. have reported that switching from oral bisoprolol to the transdermal patch was safe and effective in patients with chronic heart failure [23]. Only a few reports have described the effect of a transdermal patch on clinical outcomes after cardiac surgery; however, our data showed that the transdermal patch could be safely administered similar to oral bisoprolol in this group of patients. 
The perioperative administration of TBP scores over oral bisoprolol because: (1) The plasma concentration-time profile of the transdermal patch is more stable than that of oral $\beta$-blockers and prevents rapid changes in blood pressure and heart rate. (2) A transdermal patch can be easily removed if hypotension or bradycardia occurs. Although the blood level of a transdermal patch can be sustained after removal, it may be possible to remove the patch before the blood concentration reaches the maximum blood level as the absorption of $\beta$-blocker in patients with the patch is slower compared with oral type [9, 24]. (3) Heart failure-induced edema of the intestines can block the gastrointestinal absorption of orally administered $\beta$-blockers. Some authors have reported that use of cardiopulmonary bypass was associated with gastrointestinal injury and edema formation $[25,26]$. The percutaneous absorption of bisoprolol incorporated in the transdermal patch may overcome this drawback. (4) Transdermal bisoprolol is safely administered even in patients with impaired swallowing.

\section{Study limitations}

Limitations of our study are: (1) this was a single-center study involving a small sample size. (2) Patients included in this study underwent various types of surgeries, which could affect the incidence of POAF, although no intergroup difference was observed in the types of surgeries performed. (3) The retrospective study design serves as a drawback. Randomized control studies are required to confirm the superiority of the transdermal patch over oral bisoprolol.

\section{Conclusions}

The incidence of new-onset POAF in patients administered TBP was lower than that in patients administered oral bisoprolol after cardiac and/or thoracic aortic surgery. The TBP patch is an effective and safe $\beta$-blocker drug delivery system to prevent POAF.

\section{Compliance with ethical standards}

Conflict of interest The authors declare that they have no conflict of interest.

\section{References}

1. Akaishi M. Intravenous infusion of ultra-short-acting $\beta$-blocker for postoperative atrial fibrillation is the one of choice. Circ J. 2012;76:1083-4.

2. Chua SK, Shyu KG, Lu MJ, Hung HF, Cheng JJ, Lee SH, et al. Association between renal function, diastolic dysfunction, and postoperative atrial fibrillation following cardiac surgery. Circ J. 2013;77:2303-10.

3. Aranki SF, Shaw DP, Adams DH, Rizzo RJ, Couper GS, Vander Vilet M, et al. Predictors of atrial fibrillation after coronary artery surgery. Current trends and impact on hospital resources. Circulation. 1996;94:390-7.

4. Saito A, Hirahara N, Motomura N, Miyata H, Takamoto S. Current status of cardiovascular surgery in Japan, 2013 and 2014: a report based on the Japan Cardiovascular Surgery database 3. Coronary artery bypass surgery. Gen Thorac Cardiovasc Surg. 2018;66:8-12.

5. January CT, Wann LS, Alpert JS, Calkins H, Cigarroa JE, Cleveland JC Jr, et al. 2014 AHA/ACC/HRS guideline for the management of patients with atrial fibrillation: executive summary: a report of the American College of Cardiology/American Heart Association TaskForce on practice guidelines and the Heart Rhythm Society. Circulation. 2014;130:2071-104.

6. Sousa-Uva M, Head SJ, Milojevic M, Collet JP, Landoni G, Castella M, et al. 2017 EACTS Guidelines on perioperative medication in adult cardiac surgery. Eur J Cardiothorac Surg. 2018;53:5-33.

7. JCS Joint Working Group. Guidelines for pharmacotherapy of atrial fibrillation. Circ J. 2014;78:1997-2021.

8. Hara T, Yagi S, Akaike M, Sata M. Transdermal patch of bisoprolol for the treatment of hypertension complicated with aortic dissection. Int J Cardiol. 2015;198:220-1.

9. Sairaku A, Nakano Y, Shiode N, Suenari K, Oda N, Ono K, et al. Head-to-head comparison of the heart rate variability between the bisoprolol transdermal patch and bisoprolol fumarate tablet. Cardiovasc Ther. 2018;36:e12325.

10. Godo S, Kawazoe Y, Ozaki H, Fujita M, Kudo D, Nomura R, et al. Switching therapy from intravenous landiolol to transdermal bisoprolol in a patient with thyroid storm complicated by decompensated heart failure and gastrointestinal dysfunction. Intern Med. 2017;56:2603-9.

11. Matsuoka H, Kuwajima I, Shimada K, Mitamura H, Saruta T. Comparison of efficacy and safety between bisoprolol transdermal patch (TY-0201) and bisoprolol fumarate oral formulation in Japanese patients with grade I or II essential hypertension: randomized, double-blind, placebo-controlled study. J Clin Hyopertens (Greenwich). 2013;11:806-14.

12. Takahashi S, Katayama K, Watanabe M, Kodama H, Taguchi T, Kurosaki T, et al. Preoperative tissue doppler imaging-derived atrial conduction time predicts postoperative atrial fibrillation in patients undergoing mitral valve surgery for mitral valve regurgitation. Circ J. 2016;80:101-9.

13. Kaireviciute D, Aidietis A, Lip GY. Atrial fibrillation following cardiac surgery: clinical features and preventative strategies. Eur Heart J. 2009;30:410-25.

14. Banach M, Goch A, Misztal M, Rysz J, Jaszewski R, Goch JH. Predictors of paroxysmal atrial fibrillation in patients undergoing aortic valve replacement. J Thorac Cardiovasc Surg. 2007;134:1569-76.

15. Yokota J, Nishi H, Sekiya N, Yamada M, Takahashi T. Atrial fibrillation following aortic valve replacement: impact of perioperative use of intravenous $\beta$-blocker. Gen Thorac Cardiovasc Surg. 2017;65:194-9.

16. Maniar PB, Balcetyte-Harris N, Tamis JE, Steinberg JS. Intravenous versus oral beta-blockers for prevention of post-CABG atrial fibrillation in high-risk patients identified by signal-averaged ECG: lessons of a pilot study. Card Electrophysiol Rev. 2003; 7:158-61.

17. Jalalian R, Ghafari R, Ghazanfari P. Comparing the therapeutic effects of carvedilol and metoprolol on prevention of atrial fibrillation after coronary artery bypass surgery, a double-blind study. Int Cardiovasc Res J. 2014;8:111-5. 
18. DiNicolantonio JJ, Beavers CJ, Menezes AR, Lavie CJ, O'Keefe $\mathrm{JH}$, Meier P, et al. Meta-analysis comparing carvedilol versus metoprolol for the prevention of postoperative atrial fibrillation following coronary artery bypass grafting. Am J Cardiol. 2014;113:565-9.

19. Hillis LD, Smith PK, Anderson JL, Bittl JA, Bridges CR, Byrne JG, et al. 2011 ACCF/AHA guideline for coronary artery bypass graft surgery: a report of the American College of Cardiology Foundation/American Heart Association Task Force on Practice Guidelines. Circulation. 2011;124:e652-735.

20. Sakamoto A, Kitakaze M, Takamoto S, Namiki A, Kasanuki H, Hosoda S, JL-KNIGHT study group. Landiolol, an ultra-shortacting $\beta 1$-blocker, more effectively terminates atrial fibrillation than diltiazem after open heart surgery: prospective, multicenter, randomized, open-label study (JL-KNIGHT Study). Circ J. 2012;76:1097-101.

21. Sezai A, Minami K, Nakai T, Hata M, Yoshitake I, Wakui S, et al. Landiolol hydrochloride for prevention of atrial fibrillation after coronary artery bypass grafting: new evidence from the PASCAL trial. J Thorac Cardiovasc Surg. 2011;141:1478-87.

22. Sezai A, Osaka S, Yaoita H, Ishii Y, Arimoto M, Hata H, et al. Safety and efficacy of landiolol hydrochloride for prevention of atrial fibrillation after cardiac surgery in patients with left ventricular dysfunction: prevention of atrial fibrillation after cardiac surgery with landiolol hydrochloride for left ventricular dysfunction (PLATON) trial. J Thorac Cardiovasc Surg. 2015;150:957-64.

23. Momomura S, Saito Y, Yasumura Y, Yamamoto K, Sakata Y, Daimon $\mathrm{M}$, et al. Efficacy and safety of switching from oral bisoprolol to transdermal patch in Japanese patients with chronic heart failure. Circ J. 2018;82:141-7.

24. Drago S, Imboden R, Schlatter O, Buylaert M, Krähenbühl S, Drewe J. Pharmacokinetics of transdermal etofenamate and diclofenac in healthy volunteers. Basic Clin Pharmacol Toxicol. 2017;121:423-9.

25. Sinclair DG, Haslam PL, Quinlan GJ, Pepper JR, Evans TW. The effect of cardiopulmonary bypass on intestinal and pulmonary endothelial permeability. Chest. 1995;108:718-24.

26. Tokufuji M, Stahl GL, Metais C, Tomita M, Agah A, Bianchi C, et al. Mesenteric dysfunction after cardiopulmonary bypass: role of complement C5a. Ann Thorac Surg. 2000;69:799-807.

Publisher's Note Springer Nature remains neutral with regard to jurisdictional claims in published maps and institutional affiliations. 